\title{
High Voltage Measurements by sampling technique
}

\author{
Mohamed Agazar LNE ${ }^{1}$ \\ ${ }^{1}$ Laboratoire National de Métrologie et d'Essais, 29 avenue Roger Hennequin 78197 Trappes, France
}

\begin{abstract}
A new method, based on the sampling technique, has been developed in LNE for the calibration of high voltage dividers and high voltage transformers for frequencies from $15 \mathrm{~Hz}$ to $1000 \mathrm{~Hz}$. The divider under calibration is compared to a high voltage standard; the output voltages are sampled by two sampling voltmeters; the ratio and the phase shift of the divider under calibration are calculated with a Discrete Fourier Transformer algorithm. This method is characterized for voltage up to $300 \mathrm{kV}$ at $50 \mathrm{~Hz}-60 \mathrm{~Hz}$ frequency range, for voltage up to $100 \mathrm{kV}$ at $60 \mathrm{~Hz}-1000 \mathrm{~Hz}$ frequency range and for voltage up to $30 \mathrm{kV}$ at $15 \mathrm{~Hz}-50 \mathrm{~Hz}$ frequency range. The uncertainties are less than $0,002 \%$ for the ratio and less than $20 \mu \mathrm{rad}$ for phase shift at 50 $\mathrm{Hz}-60 \mathrm{~Hz}$ frequency range. At other frequencies they are less than $0,005 \%$ for the ratio and less than $50 \mu \mathrm{rad}$ for the phase shift.
\end{abstract}

\section{Présentation du manuscrit}

Dans un souci d'optimisation de leur consommation d'énergie ou plus simplement parce qu'ils utilisent de la haute tension pour leurs activités, certaines industries souhaitent connaître avec la meilleure exactitude possible la valeur de cette tension. A titre d'exemple, dans une plateforme de production d'électricité de $750 \mathrm{MW}$, une erreur de $0,1 \%$ sur la mesure de la tension de $400 \mathrm{kV}$ implique un écart de 0,75 MW, soit l'électricité nécessaire pour alimenter 220 foyers. Les fabricants travaillent constamment pour réduire les erreurs de leurs diviseurs de haute tension afin de les rendre négligeables.

Des diviseurs de tension avec des erreurs inférieures à $0,005 \%$ pour l'erreur de rapport et inférieures à $80 \mu \mathrm{rad}$ pour le déphasage ont été récemment commercialisés. La qualification et l'étalonnage de ces diviseurs nécessitent la disposition de méthodes offrant des incertitudes meilleures que les erreurs annoncées par ces fabricants.

Pour répondes à ces nouvelles exigences, le LNE a mis en place une nouvelle méthode d'étalonnage basée sur un échantillonnage et une conversion analogique numérique des signaux. Le traitement des échantillons est effectué avec une Transformée de Fourier Discrète offrant la possibilité d'accéder non seulement à l'amplitude et à la phase du fondamental mais également à l'amplitude et à la phase de l'ensemble des harmoniques.

\section{Principe de mesure}

La figure 1 illustre le principe de mesure par la technique d'échantillonnage d'un transformateur de tension.

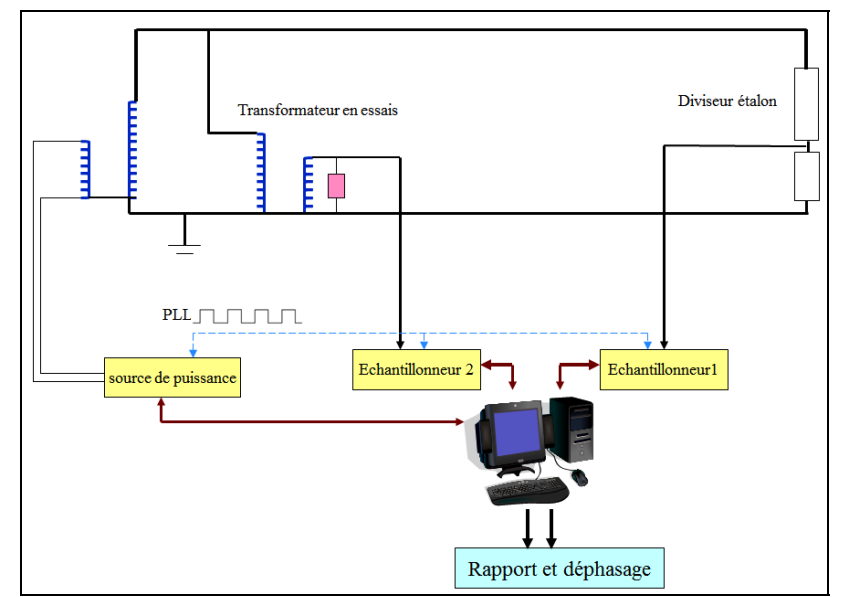

Figure 1: Principe d'étalonnage d'un diviseur HT (cas d'un transformateur de tension)

Le diviseur en étalonnage est comparé à un diviseur haute tension étalon de rapport connu $\mathrm{K}_{\mathrm{e}}$ et de déphasage connu $\varphi_{\mathrm{e}}$. Les deux tensions en sortie des deux diviseurs sont échantillonnées par deux échantillonneurs. Les signaux échantillonnés sont traités par un algorithme afin d'extraire le rapport $K_{\text {éch }}$ et le déphasage $\varphi_{\text {éch }}$ entres ces deux tensions.

Le rapport $K_{\mathrm{x}}$ et le déphasage $\varphi_{\mathrm{x}}$ du diviseur en étalonnage sont donnés respectivement par les formules (1) et (2):

$$
\begin{aligned}
& K_{x}=K_{e} \times K_{e ́ c h} \\
& \varphi_{x}=\varphi_{e}+\varphi_{e ́ c h}
\end{aligned}
$$

Mesurer $\mathrm{K}_{\mathrm{x}}$ revient donc à mesurer le rapport des tensions échantillonnées par les deux échantillonneurs.

\footnotetext{
a Email de correspondance de l'auteur : auteur@email.org
} 
Mesurer $\varphi_{\mathrm{x}}$ revient donc à mesurer le déphasage entre les tensions échantillonnées par les deux échantillonneurs.

\section{L'échantillonnage}

\subsection{Principe}

Le traitement des échantillons est fait par transformée de Fourier. Elle permet d'accéder à l'amplitude et à la phase du fondamental mais également à l'amplitude et à la phase de l'ensemble des harmoniques.

Le mesurande étant défini pour des signaux sinusoïdaux, seules les informations concernant le fondamental sont utiles. Dans ce cas, l'évaluation de l'amplitude et de la phase des signaux consiste à déterminer le niveau maximum de la raie observée en module de la transformée de Fourier et à considérer la phase correspondante.

En pratique, le nombre d'opérations réalisables ne pouvant être infini, un algorithme de Transformée de Fourier Discrète est appliqué (l'algorithme de FFT). Dans ce cas, la représentation fréquentielle se trouve échantillonnée et il est nécessaire d'assurer la coïncidence entre le maximum de la raie et un point d'échantillonnage comme le montre la figure 2 .

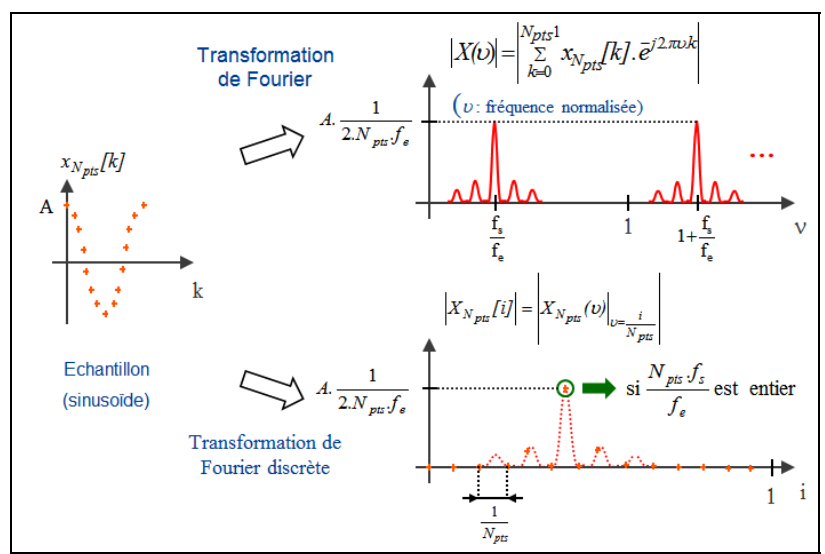

Figure 2: Coïncidence entre le maximum de la raie et un point d'échantillonnage.

Pour cela, la condition de la formule (3) doit être respectée:

$$
f_{e}=\frac{N_{p t s}}{N_{p e r}} f_{s}
$$

Avec:

$\mathrm{f}_{\mathrm{s}}$ : fréquence du signal de mesure.

$\mathrm{f}_{\mathrm{e}}$ : fréquence d'échantillonnage.

$\mathrm{N}_{\text {pts }}$ : nombre d'échantillons.

$\mathrm{N}_{\text {per }}$ : nombre de périodes.

Cette condition requiert la maîtrise du rapport entre la fréquence d'échantillonnage et la fréquence du signal.
Une boucle à verrouillage de phase PLL (Phase Locked Loop) est conçue pour garantir cette condition.

\subsection{La boucle à verrouillage de phase (PLL)}

La boucle à verrouillage de phase permet de générer un signal de cadencement de fréquence $f_{e}$ en phase avec la fréquence fondamentale $\mathrm{f}_{\mathrm{s}}$ du signal de mesure.

La PLL utilisée pendant les étalonnages permet de multiplier le signal de mesure par un multiple de deux (128, 256,512 ou 1024). La relation (3) est vérifiée lorsque le nombre de points d'échantillonnage est égal à un multiple de deux (1024 points par exemple).

La PLL peut être associée à des diviseurs de fréquence pour ne pas dépasser les fréquences d'échantillonnage admises par les deux échantillonneurs, (voir paragraphe suivant).

\subsection{Les échantillonneurs}

Les deux échantillonneurs utilisés peuvent autoriser des fréquences d'échantillonnage de $0,2 \mathrm{~Hz}$ (avec une résolution de 28 bits) jusqu'à $100 \mathrm{kHz}$ (avec une résolution de 16 bits). Le temps d'intégration est paramétrable entre $500 \mathrm{~ns}$ et $1 \mathrm{~s}$ par pas de $100 \mathrm{~ns}$. La tension maximale admise est de l'ordre de $10 \mathrm{~V}$. Pour les tensions supérieures, des diviseurs inductifs de tensions étalons peuvent être ajoutés entre la sortie des diviseurs et les échantillonneurs, leurs impédances d'entrée ne doivent pas engendrer une influence significative sur le résultat de mesure.

Pour effectuer un échantillonnage sans erreurs, une résolution d'au moins 18 bits est respectée. Cette caractéristique est utilisée pour obtenir un effet de moyenne plus important et limiter ainsi les erreurs de quantification et la dispersion des mesures. Elles sont ainsi rendues inférieures à $2 \cdot 10^{-6}$ pour le rapport et inférieures à $2 \mu \mathrm{rad}$ pour le déphasage.

Pour rester conforme aux formules (1) et (2) la caractérisation des deux échantillonneurs est effectuée en rapport (rapport entre le premier échantillonneur et le deuxième) et en différence de déphasage (différence de phase entre le premier échantillonneur et le deuxième). Cette caractérisation est réalisée par comparaison à un diviseur inductif étalon sur le rapport 1 et 0,5 .

Pour la mesure des rapports, les erreurs d'échantillonnage peuvent être donc maitrisées et corrigées avec des incertitudes inferieures à $2.10^{-6}$ pour le rapport. Comme le montre la figure 3 , pour un signal de $50 \mathrm{~Hz}$, les erreurs sont inférieures à $2.10^{-6}$ pour des fréquences d'échantillonnage inférieures à $6 \mathrm{KHz}$ et pour un temps d'intégration suffisamment grand.

Pour les mesures de déphasage, tout élément du dispositif à l'origine d'un décalage temporel entre les signaux est source d'erreur sur la mesure du déphasage. 
L'échantillonnage des signaux est commandé par un signal PLL externe simultané sur les deux échantillonneurs. Cependant, il apparaît de manière systématique que le temps de réponse des échantillonneurs diffère d'un appareil à l'autre. Cet écart est donc à l'origine d'une erreur systématique sur la mesure du déphasage. Celle-ci est évaluée à $(+0,17 \pm 0,01) \mu \mathrm{rad} / \mathrm{Hz}$ (voir figure 4).

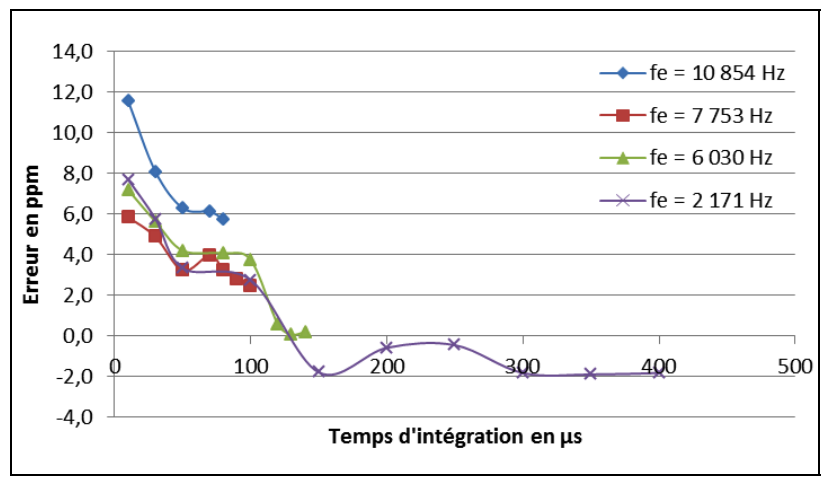

Figure 3: Erreur de rapport en fonction de $\mathrm{f}_{\mathrm{e}}, \mathrm{f}_{\mathrm{s}}=50 \mathrm{~Hz}, 5 \mathrm{~V}$

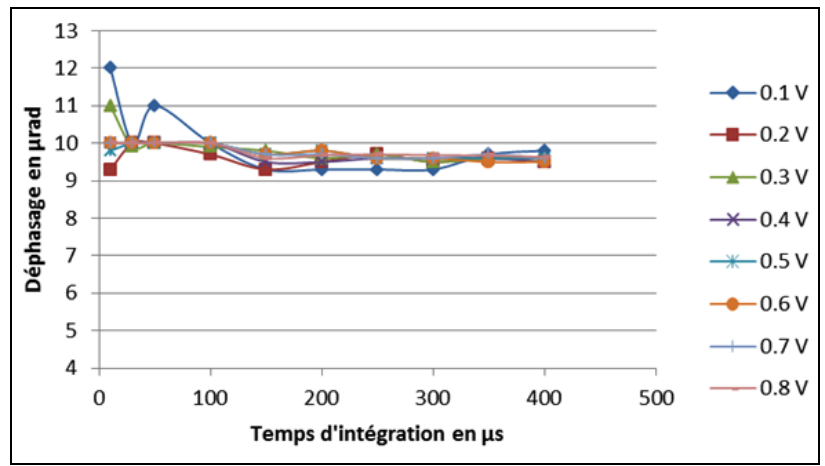

Figure 4: Déphasage en fonction de la tension, $\mathrm{f}_{\mathrm{s}}=50 \mathrm{~Hz}, 5 \mathrm{~V}$ $\mathrm{f}_{\mathrm{e}}=2,2 \mathrm{kHz}$.

\section{Les diviseurs étalons HT}

\subsection{La résistance haute tension $100 \mathrm{kV}$}

La résistance Haute Tension a été construite pour servir de réducteur exact des hautes tensions alternatives aux fréquences industrielles et spécialement conçue pour avoir des caractéristiques bien déterminées en courant alternatif. La valeur $\mathrm{R}$ de la résistance est choisie égale à $4 \mathrm{M} \Omega$ afin d'avoir un compromis entre les exigences de faible échauffement, de faible consommation et de faible déphasage :

- Le coefficient de température mesuré en courant continu est de $\left(+4,3 \cdot 10^{-6} \pm 0,2\right) \cdot 10^{-6} \cdot \mathrm{R} /{ }^{\circ} \mathrm{C}$.

- L'effet de l'échauffement de l'huile correspond à $5.10^{-6} \cdot \mathrm{R} /\left(\mathrm{kV}^{2} \cdot \mathrm{min}\right) \cdot 10^{4}$ obtenue avec une incertitude sur la résistance de $3,3 \cdot 10^{-6} . \mathrm{R}$.

- La variation de la température du fil conducteur est proportionnelle au carré de la tension appliquée avec un coefficient de $3,9 \cdot 10^{-4}{ }^{\circ} \mathrm{C} / \mathrm{kV}^{2}$. Ce dernier appliqué au coefficient de température de $4,3.10^{-6} \mathrm{R} /{ }^{\circ} \mathrm{C}$ donne un coefficient de tension de la résistance égal à $(16,8 . \pm 1,0) \cdot 10^{-10} \cdot \mathrm{R} / \mathrm{kV}^{2}$.

- La variation de la résistance série en fonction de la fréquence est obtenue en basse tension à l'aide d'un banc à échantillonnage et par comparaison à un diviseur inductif. Les incertitudes de mesure sont inférieures à $1.10^{-5} . \mathrm{R}$ pour des fréquences entre $20 \mathrm{~Hz}$ et $1000 \mathrm{~Hz}$.

- Le déphasage de la résistance est mesuré dans la configuration de l'essai parce que sa constante de temps dépend de l'environnement malgré le dispositif d'écran qu'elle contient. D'une façon générale, la proximité de toute masse conductrice reliée à la borne haute tension de la résistance a pour effet de provoquer une variation négative de la constante de temps, tandis que toute masse conductrice reliée à la masse à pour effet de provoquer une variation positive de la constante de temps. Les mesures sont effectuées en basse tension à l'aide d'un pont de mesure de capacité étalonné sur toute la plage de fréquence entre $20 \mathrm{~Hz}$ à $1000 \mathrm{~Hz}$.

\subsection{Les diviseurs capacitifs et la remontée}

Les diviseurs capacitifs sont utilisés lorsque les tensions de mesures dépassent les tensions admises par la résistance $\mathrm{HT}$ ou lorsque l'étalonnage est réalisé sur le site client. Trois diviseurs capacitifs peuvent être utilisés, le premier de rapport 1000 est utilisé quand la tension est inférieure à $10 \mathrm{kV}$, le deuxième de rapport 10000 est utilisé quand la tension est inférieure à $100 \mathrm{kV}$ et le troisième de rapport 30000 est utilisé quand la tension est inférieure à $300 \mathrm{kV}$.

Ces diviseurs capacitifs, dont le coefficient de tension est faible, sont étalonnés dans un processus de remontée à partir d'un étalonnage fait à $1000 \mathrm{~V}$ sur le premier diviseur capacitif. Cet étalonnage est effectué par comparaison à un diviseur inductif étalon de rapport 1000. Ce processus de remontée est réalisé par l'intermédiaire de transformateurs de tension étalonnés.

Les coefficients de température des diviseurs capacitifs sont maitrisés. Une correction est appliquée si nécessaire lorsqu'il y a des différences significatives entre la température de l'étalonnage du diviseur capacitif lui même et la température de son utilisation pendant la mesure de l'objet en étalonnage.

\section{Les étages adaptateurs d'impédance}

L'impédance d'entrée des échantillonneurs, qui est constituée d'une résistance de $10 \mathrm{G} \Omega$ en parallèle avec une capacité de $140 \mathrm{pF}$, se manifeste aux fréquences hautes à travers une erreur sur le rapport et sur le déphasage. Elle est plus significative quand l'impédance de sortie du diviseur en étalonnage est de forte valeur. Une correction, avec une incertitude associée non significative, peut être appliquée pour corriger les mesures effectuées aux fréquences basses. Pour les fréquences hautes cette incertitude devient prépondérante. 
Il a été décidé de concevoir des étages adaptateurs d'impédance de rapport 1 et de déphasage proche de zéro.

Le schéma de principe des étages d'entrée correspond à un montage de type amplificateur d'instrumentation (entrée différentielle). La mise en place des deux amplificateurs de garde associés à un montage triaxial en entrée ont permis d'une part d'éliminer l'influence des pertes dans les câbles, d'autre part, de constituer un dispositif de garde externe.

La contribution des étages adaptateurs d'impédance dans l'incertitude de mesure est négligeable dans la mesure où ils peuvent être associés aux deux échantillonneurs pendant leur étalonnage.

\section{Le logiciel de mesure}

Le logiciel de mesure est développé sur l'environnement Labview qui permet, en plus de l'échantillonnage et le traitement des échantillons, de réaliser de façon complètement automatique les séries de mesures.

\section{Exemple de résultats de mesures}

Les graphes 5 et 6 donnent les résultats de mesures trouvés pendant les essais de validation. Les mesures sont faites sur un transformateur de $15 \mathrm{kV} / 100 \mathrm{~V}$ par comparaison aux différents étalons. Les résultats de mesures montrent un bon recoupement entre les différentes mesures $\left(2.10^{-5}\right.$ pour le rapport et $20 \mu \mathrm{rad}$ pour le déphasage) bien que les conditions de mesure ne soient pas respectées rigoureusement (température, boucles de masse, connexions haute tension, distance entre objets, tension de mesure).

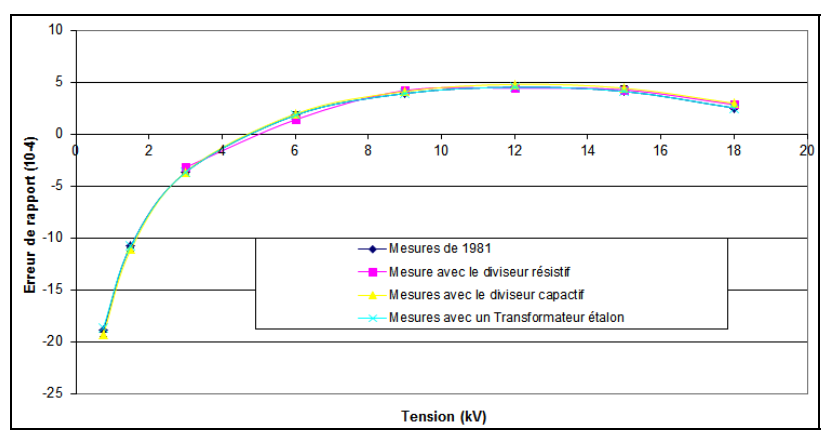

Figure 5: Mesures de l'erreur de rapport, $\mathrm{f}_{\mathrm{s}}=50 \mathrm{~Hz}, \mathrm{f}_{\mathrm{e}}=6,4 \mathrm{kHz}$

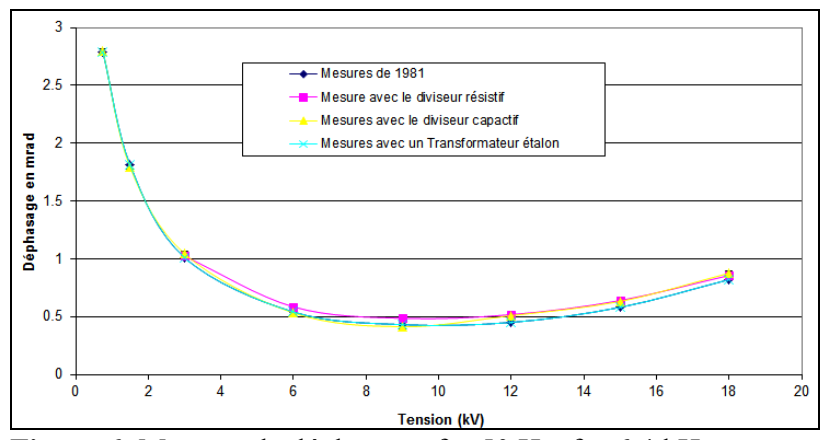

Figure 6: Mesures de déphasage, $\mathrm{f}_{\mathrm{s}}=50 \mathrm{~Hz}, \mathrm{f}_{\mathrm{e}}=6,4 \mathrm{kHz}$

\section{Conclusion}

Cette nouvelle méthode va permettre au LNE de disposer d'un nouveau banc pour l'étalonnage des diviseurs en haute tension alternative. Elle assurera une amélioration de l'exactitude des résultats ainsi que les incertitudes de mesure. Elle permettra d'étendre les mesures aux fréquences basses jusqu'à $15 \mathrm{~Hz}$ à $30 \mathrm{kV}$ et aux fréquences hautes jusqu'à $1000 \mathrm{~Hz}$ à 100 kV .

\section{Références}

1. P.Espel « Développement d'un wattmètre numérique à échantillonnage » Revue Française de métrologie, 12, 2007, 3-12.

2. R.L.Swerlein « Precision AC Voltage Measurements Using Digital Sampling Techniques » HEWLETTPACKARD JOURNAL, avril 1989. 